\title{
CONTRIBUTION
}

\section{Special Issue on 4D Flow MRI in Magnetic Resonance in Medical Sciences}

\begin{abstract}
Michael Markl ${ }^{1,2^{*}}$
4D flow MRI has emerged as a versatile imaging technique for the in vivo measurement of cardiac and vascular 3D hemodynamics. The feasibility of 4D flow MRI was initially demonstrated by $3 \mathrm{D}$ visualizations of dynamic changes in blood flow patterns and their associations with different cardiovascular abnormalities, e.g., the impact of heart valve disease on deranged flow in the aorta and pulmonary artery. The continued evolution of 4D flow MRI over the past two decades comprised a shift from qualitative imaging of flow patterns toward a focus on quantitative analysis workflows, including standardized flow parameters (peak velocity, net flow, etc.) and also advanced hemodynamics measures, such as wall shear stress, to investigate the role of abnormal hemodynamics at play in vessel wall remodeling. In tandem with the development of reliable quantitative imaging parameters, there has been a focus on making 4D flow MRI faster and easier to use in the clinical setting. Novel acceleration techniques such as compressed sensing, and more recently, deep learning concepts, have reduced scan times to less than 5 minutes, a significant improvement from imaging sessions that, once regularly, take 20-30 minutes. In addition, interpretation of 4D flow MRI no longer requires a dedicated research team. Post-processing workflows have been streamlined; visualization and analysis of data have become more straightforward and are now readily integrated with commercially available software solutions for cardiovascular image analysis.

This special issue on 4D flow MRI in the journal of Magnetic Resonance in Medical Sciences documents this progress in 4D flow MRI. The individual contributions to this special issue provide strong testament to the translational potential of 4D flow MRI from an imaging tool initially relegated to the research arena to a versatile technique for clinical evaluation of abnormal cardiac and vascular 3D flow dynamics in application areas throughout the human circulatory system. The editors and authors should be congratulated for providing a comprehensive and highly timely overview of the current state of the art in 4D flow imaging.
\end{abstract}

'Department of Radiology, Northwestern University, Feinberg School of Medicine, Chicago, IL, USA

'Department of Biomedical Engineering, Northwestern University, McCormick

School of Engineering, Evanston, IL, USA
This work is licensed under a Creative Commons Attribution-NonCommercial-NoDerivatives International License.

(C2022 Japanese Society for Magnetic Resonance in Medicine 